\title{
Effect of dexmedetomidine on hippocampal neuron development and BDNF-TrkB signal expression in neonatal rats
}

This article was published in the following Dove Press journal:

Neuropsychiatric Disease and Treatment

9 December 2016

Number of times this article has been viewed

Jie Lv

Wei Ou

Xiao-Hua Zou

Yin Yao

Jin-Li Wu

Department of Anesthesia, Guizhou Medical University Affiliated Hospital, Guiyang, People's Republic of China

Correspondence: Wei Ou Department of Anesthesia, Guizhou Medical University Affiliated Hospital, 4 Beijing Road, Guiyang 55000I, People's Republic of China

Tel +86 I809605 7766

Email ouweigzmu@foxmail.com
Abstract: The study aimed to explore the effect of dexmedetomidine (DEX) on hippocampal neuron development process and on molecular expression of brain-derived neurotrophic factor (BDNF)-tyrosine receptor kinase B (TrkB) signaling pathway in neonatal rats. The hippocampal neuron cells were isolated from newborn neonatal rats and cultured in vitro. One control group and three treated groups with 1,10 , and $100 \mu \mathrm{mol} / \mathrm{L}$ DEX were used for the study. Cell activity and apoptosis were detected by the MTT and terminal deoxynucleotidyl transferase-mediated biotinylated uridine triphosphate (UTP) nick end labeling assays. The synaptophysin (SYN) and postsynaptic density 95 (PSD95) were detected by quantitative polymerase chain reaction. There was no difference in the viability of neuron cells among the different dose groups of DEX and the control group during days $2-10(P>0.05)$. Compared to the control group, there was no significant difference $(P>0.05)$ in the expressions of SYN and PSD95 in the groups treated with 1 and $10 \mu \mathrm{mol} / \mathrm{L}$ DEX, whereas significant difference in the expression was observed in the group treated with $100 \mu \mathrm{mol} / \mathrm{L}$ DEX $(P<0.01)$. Compared with the control group, the expression of BDNF was significantly upregulated $(P<0.05)$ in the group treated with $100 \mu \mathrm{mol} / \mathrm{L}$ DEX. There were no significant differences in TrkB expression among the four groups. The expression of p-N-methyl-D-aspartate receptor increased with an increase in the concentration of DEX; however, only the high dose revealed a significant upregulation compared with the control group. The neuroprotective effect of DEX may be achieved by upregulating the expression of BDNF and phosphorylation level of N-methyl-D-aspartate receptor.

Keywords: dexmedetomidine, hippocampal neuron, development, BDNF-TrkB

\section{Introduction}

As an important member of the nerve factor family, brain-derived neurotrophic factor (BDNF) regulates and maintains the development and function of the nervous system by promoting the proliferation and differentiation of nerve cells. Synapse is an important way of transferring information between neurons, and its structure consists of the front membrane, the gap, and the posterior membrane. Neurons form complex neural networks in the form of synapses and play an important role in the physiological and pathological processes of the central nervous system. After the specific binding between BDNF and tyrosine receptor kinase B (TrkB), the conformation changes, which lead to the phosphorylation of downstream key kinase, promote the expression of BDNF in neurons and dendrites, and activate the BDNF-TrkB signaling pathway. BDNF is first transported to the presynaptic neurons where it displays its regulatory function. Therefore, BDNF plays an important role in presynaptic regulation. ${ }^{1-3}$ 
Dexmedetomidine (DEX) is a type of $\alpha_{2}$-adrenergic receptor agonist with the main ingredient DEX hydrochloride injection, the $\alpha_{2}$-adrenergic receptor affinity is eight times that of clonidine. ${ }^{46} \mathrm{DEX}$ is widely used as an auxiliary drug in clinical anesthesia. With the application of general anesthesia in clinics, increasing attention has been given to central toxic effects of general anesthetics in recent years, especially on the development of neurons, which is of great significance in infants. ${ }^{7}$

This study explored the effect of DEX on the expression of genes involved in the neonatal hippocampal neuron development and on the expression of molecules involved in the BDNF-TrkB signaling pathway by isolating and culturing neurons in the hippocampus of neonatal rats. The synaptophysin (SYN) was distributed in the presynaptic membrane and postsynaptic density 95 (PSD95) was located in the postsynaptic structure. These synapse-specific protein markers may directly or indirectly reflect the synaptic activity, morphological changes, and the biological function of the synapse. ${ }^{6,8}$ We analyzed the activity and apoptosis of neurons in the hippocampus of neonatal rats, analyzed the effect of DEX on BDNF-TrkB signal pathway, and investigated the molecular mechanism of the biological effects that DEX exerts through BDNF-TrkB signaling pathway.

\section{Materials and methods Experimental animals}

Specific pathogen-free (SPF) grade Sprague Dawley pregnant rats were purchased from Shanghai Silaike Experimental Animals Inc. (Shanghai, People's Republic of China). The mean $( \pm$ standard deviation) rat weight was $250( \pm 30 \mathrm{~g})$, and they were fed with standard pellets in standard animal cages (three animals per cage). The feeding room had good ventilation and natural lighting. Room temperature was maintained at $18^{\circ} \mathrm{C}-25^{\circ} \mathrm{C}$. The primary hippocampal neurons were isolated from neonatal rats within 24 hours after delivery. The study protocol was approved by the Institutional Review Board of the Guizhou Medical University Affiliated Hospital. All animal handling procedures were carried out in accordance with the protocols of the animal care guidelines of the Institutional Animal Care Committee.

\section{Main reagents and instruments}

The following reagents and chemicals were used for the experiments: Dulbecco's Modified Eagle's Medium (DMEM), fetal bovine serum, Neurobasal ${ }^{\circledR}$, and B27 additives (Thermo Fisher Scientific, Waltham, MA, USA); L-glutamine (Sigma-Aldrich Co., St Louis, MO, USA); DEX (Jiangsu Hengrui Medicine Co., Lianyungang, People's Republic of China); terminal deoxynucleotidyl transferase-mediated biotinylated uridine triphosphate (UTP) nick end labeling (TUNEL) kit (Vazyme Biotech Co., Piscataway, NJ, USA); MTT detection kit (Beijing Boosen Biological Technology Co., Beijing, People's Republic of China); RNA purification kit (QIAGEN Translational Medicine Co., Suzhou, People's Republic of China); reverse transcription kit (Applied Biosystems Co., Foster City, CA, USA); SYBR Green real-time PCR premixed liquid (Applied Biosystems Co.); ReadyPrep protein extraction kit (Bio-Rad Laboratories Inc., Hercules, CA, USA); MAP-2 monoclonal antibody (Santa Cruz Biotechnology Inc., Dallas, TX, USA); BDNF, TrkB, and glyceraldehyde 3-phosphate dehydrogenase (GAPDH) monoclonal antibody (Abcam, Cambridge, UK); p-N-methyl-D-aspartate receptor (p-NMDAR) and NMDAR-2B monoclonal antibody (CST Co., Danvers, MA, USA); horseradish peroxidase (HRP)-labeled secondary antibody (Wuhan Boster Bioengineering Limited Co., Wuhan, People's Republic of China). The following instruments were used: optical microscope (OLYMPUS BX53), $\mathrm{CO}_{2}$ incubator (Thermo Scientific Series 8000), nucleic acid quantitative instrument (Nanodrop 2000), and fluorescence quantitative PCR detection system (Applied Biosystems 7500).

\section{Isolation and culture of hippocampal neurons in neonatal rats}

The neonatal rats were sacrificed within 24 hours after delivery by cervical dislocation. Then the neonatal rats were placed in $75 \%$ ethanol for 2 minutes. We removed the skin and skull of the rats' head and took out the whole brain. With the help of an anatomical microscope, we carefully removed the olfactory bulb, septum, thalamus, and hypothalamus. We then peeled off the dural and choroid plexus and took out the hippocampus tissue under the cerebral cortex into pre-cold sterile phosphate buffered saline (PBS). The hippocampus tissue was cut into pieces with ophthalmology scissors, and then $0.25 \%$ trypsin $/ 0.02 \%$ ethylene diamine tetra acetic acid (EDTA) was added and digested at $37^{\circ} \mathrm{C}$ for 5 minutes to prepare single cell suspension. After removing the supernatant by centrifugation, the cell precipitate was suspended in DMEM complete culture medium (containing 2\% B27, 1\% glutamine, $10 \%$ fetal bovine serum), inoculated in cell culture bottle, and cultured at $37^{\circ} \mathrm{C}$ in a $5 \% \mathrm{CO}_{2}$ incubator. After adherent for $6-8$ hours, the cells were transferred to Neurobasal medium (for use immediately, containing $2 \%$ $\mathrm{B} 27,1 \%$ glutamine, and $100 \mathrm{IU} / \mathrm{mL}$ penicillin).

\section{Western blot}

After cleaning with PBS, the total protein was extracted using the protein extraction kit and the concentration was quantified by the bicinchoninic acid method. After the protein 
sample was subjected to substrate-sodium dodecyl sulfatepolyacrylamide gel electrophoresis (SDS-PAGE), the gel was dipped in the transfer buffer balance for 10 minutes and transferred to the membrane for 45-60 minutes. Then the polyvinylidene difluoride (PVDF) membrane was rinsed for 10-15 minutes with tris-buffered saline (TBS), and proper dilution of antibody (containing $1 \%$ of skim milk diluted with TBS containing Tween-20 [TBST]) was added and incubated at room temperature for 2 hours. Then the membrane was rinsed three times (each for 5-10 minutes) with TBST and was incubated with HRP-labeled secondary antibody, which was diluted with TBST containing $0.05 \%$ of skim milk $(1: 10,000)$. Again, the membrane was rinsed three times with TBST, each for 5-10 minutes. The experimental results were preserved by exposure and photographic processing. Quantity one Version 4.62 software was used to measure the gray value of molecular bands (quantitative method of strip track). On the quantitative basis, the semi-quantitative value of target protein/reference protein was used for statistical analysis.

\section{q-PCR detection}

Total RNA was extracted according to the manufacturer's instructions and concentration and purity were detected by Nanodrop 2000. According to the reverse transcription kit instructions, total RNA was reverse transcribed to cDNA. Real-time PCR was used to detect related genes. To request for target gene mRNA sequences in the NCBI database, we designed the real-time PCR primers, whose specific sequences were as follows: SYN (NM_008596) forward: CGCACCTCGGACAAGTCTC, reverse: CCCGAAGG CGAAAATAGCAAA; Dlg4 (PSD95, NM_001109752) forward: TCCGGGAGGTGACCCATTC; reverse: TTT CCGGCGCATGACGTAG. PCR reaction parameters were as follows: pre-denaturation at $94^{\circ} \mathrm{C}$ for 10 minutes, denaturation at $95^{\circ} \mathrm{C}$ for 10 seconds, annealing temperature according to the melting temperature of primer, reaction time of 20 seconds, extend at $72^{\circ} \mathrm{C}$ for 30 seconds with 40 cycles.

\section{Statistical analysis}

Mean and standard deviation (SD) were used to summarize continuous variables. One-way analysis of variance was used to determine the differences among groups. $P$-value $<0.05$ was considered to be statistically significant. All analyses were performed using SPSS software, Version 17.0.

\section{Results}

\section{Culture of hippocampal neurons in vitro}

The hippocampal neurons were separated from the rats born within 24 hours and cultured in vitro. When observed under inverted microscope, about $80 \%$ of the neurons began to stick to the wall after 5-6 hours and were circular or elliptic in shape. The cells continued to grow long after 10 hours with small bumps. After 3 days, cell volume increased with multiple extensions for shape or spindle. After 20 days of culture, nerve cells began to appear degenerated, with granularvacuolar degeneration in cytoplasm, degradation, and nuclear pyknosis. The neurons were identified by neuron markers such as MAP-2 in the cells growing on the glass slide. The MAP2-positive cells, cell body, and bumps were dyed brown. By observing the ratio of the number of MAP-2-positive cells to the total number of cells marked by Hoechst stain, its purity was found to be $90.2 \% \pm 1.7 \%$ when cultured for 7 days.

\section{Effect of DEX on the development of hippocampus neuron cells}

The animals were divided into four groups as follows: control group (Ctrl), treated group cultured with $1 \mu \mathrm{mol} / \mathrm{L}$ DEX (group 1), treated group cultured with $10 \mu \mathrm{mol} / \mathrm{L} \mathrm{DEX}$ (group 10), and treated group cultured with $100 \mu \mathrm{mol} / \mathrm{L}$ DEX (group 100). MTT and TUNEL methods were used to determine cell activity and apoptosis. The results of the MTT test showed that there was no difference in the activity and apoptosis curves of neuron cells among the different DEX-treated groups and the control group during 2-10 days $(P>0.05$; Figure 1A). Cells that grew on the glass slide were prepared and detected by fluorescence and counted by ImagePro Plus 6.0 software. There were no significant differences among the four groups with regard to the number of positive cells $(P>0.05$; Figure $1 \mathrm{~B})$.

The expressions of SYN and PSD95 were detected by q-PCR to explore the effect of DEX on the development of hippocampal neurons. Compared with the control group, the expressions of SYN and PSD95 exhibited no significant differences $(P>0.05)$ in the groups treated with 1 or $10 \mu \mathrm{mol} / \mathrm{L}$ DEX, whereas a significant difference with regard to the expression was observed in the group treated with $100 \mu \mathrm{mol} / \mathrm{L}$ DEX $(P<0.01$; Figure 2$)$.

\section{Effect of DEX on the expression of signaling molecules in the BDNF-TrkB signal pathway}

Western blot analysis was used to analyze the effect of DEX on the expression and phosphorylation level of BDNF, TrkB, and N-methyl-D-aspartate receptor (NMDA). As shown in Figure 3, the expression of BDNF was positively correlated with the appropriate concentration of DEX. Compared with the control group, the expression of BDNF was significantly upregulated in the group treated with $100 \mu \mathrm{mol} / \mathrm{L}$ DEX 

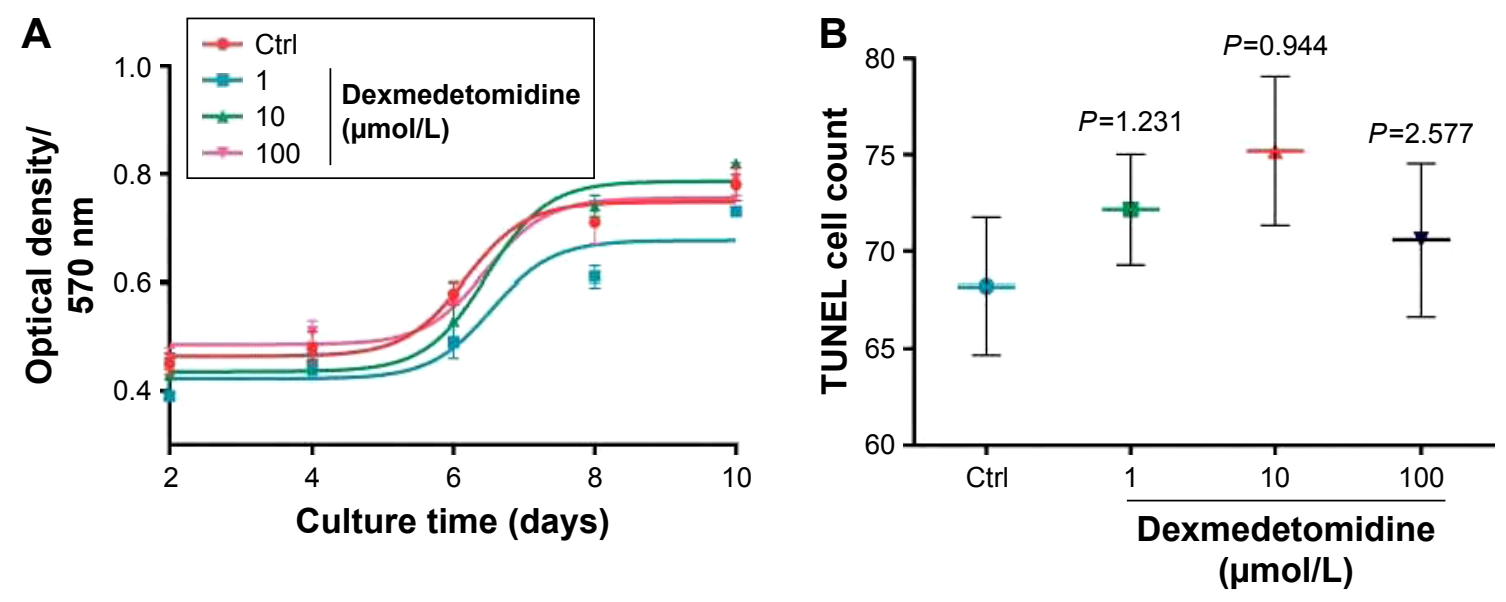

Figure I Cell activity and apoptosis were detected by MTT (A) and TUNEL (B) assays.

Abbreviations: Ctrl, control group; TUNEL, terminal deoxynucleotidyl transferase-mediated biotinylated UTP nick end labeling; UTP, uridine triphosphate.

$(P<0.05)$. There were no significant differences in TrkB expression among the four groups. The expression of p-NMDAR increased with the increasing concentration of DEX; however, only the high dose revealed a significant upregulation compared with the control group.

\section{Discussion}

General anesthetics usually act by rendering the subject unconscious and by blocking neurotransmission via exciting gamma aminobutyric acid receptor or antagonizing NMDA receptor. ${ }^{9}$ Although general anesthesia has wide application
A
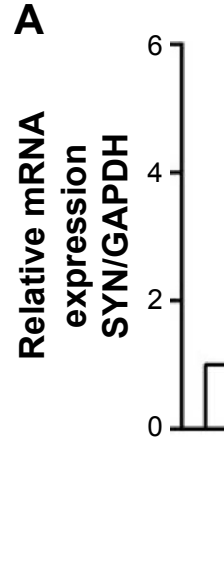

C

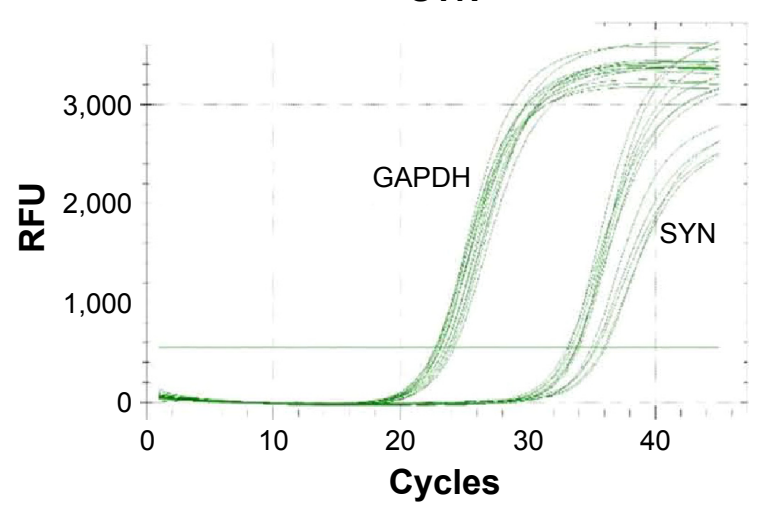

B

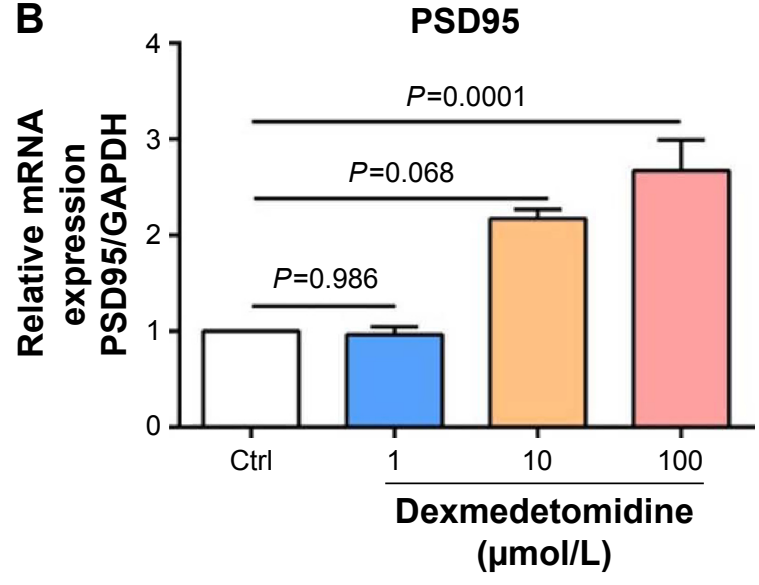

D

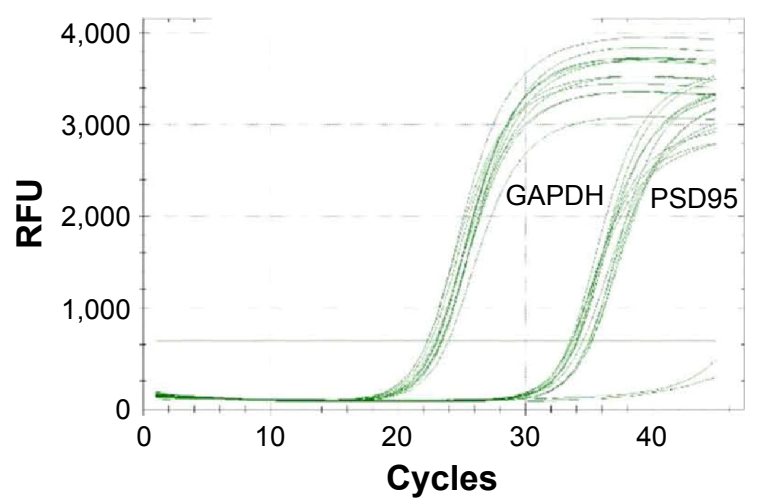

Figure 2 The relative expression and quantitative curves of SYN (A and $\mathbf{C})$ and PSD95 (B and $\mathbf{D})$ in the four groups.

Abbreviations: Ctrl, control group; GAPDH, glyceraldehyde 3-phosphate dehydrogenase; RFU, relative fluorescence unit; SYN, synaptophysin. 


\section{A}

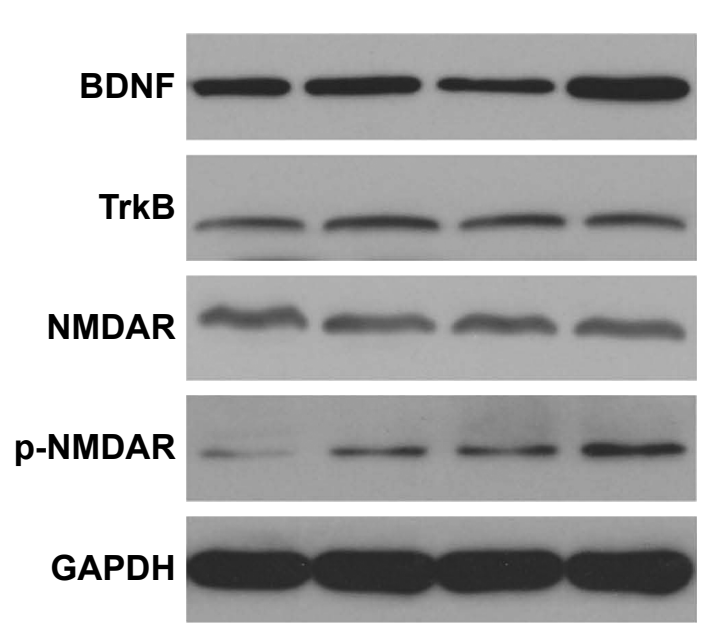

B

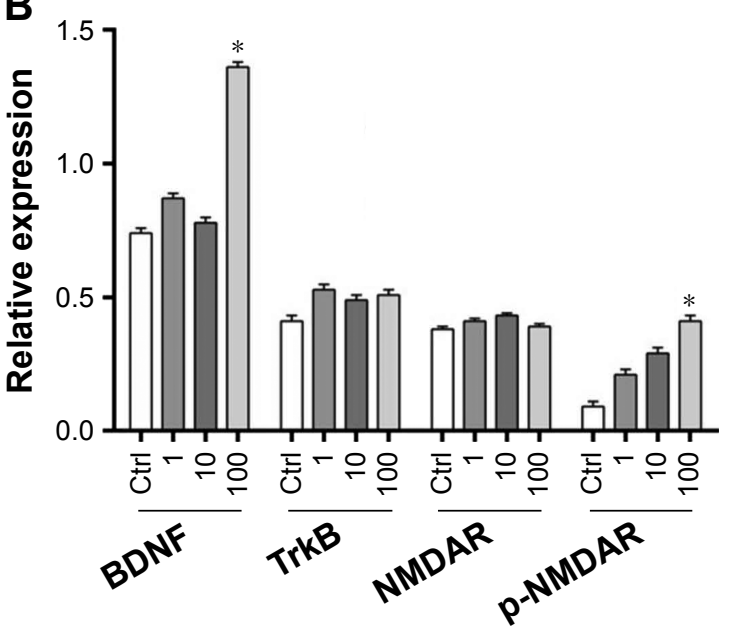

Figure 3 The expression of BDNF and TrkB in the four groups by the Western blot: (A) the electrophoretogram of Western blot; (B) the bar charts of the expression of BDNF and TrkB.

Note: *Indicates the significance compared to the control group.

Abbreviations: BDNF, brain-derived neurotrophic factor; Ctrl, control group; GAPDH, glyceraldehyde 3-phosphate dehydrogenase; NMDAR, N-methyl-D-aspartate receptor.

in clinics, further reflecting its superiority, people are increasingly concerned about its safety. The NMDA antagonists such as MK801 and ketamine might exhibit neurotoxicity. ${ }^{10}$ The combination of isoflurane and midazolam caused neuronal apoptosis in neonatal mice. ${ }^{11}$ These reports led to concerns about safety of ketamine in pediatric anesthesia. Previously, anesthesiology considered that by avoiding hypoxia of brain tissue in the anesthesia process, one could reduce the influence of anesthetic drugs on the intelligence development of infants. ${ }^{12}$ However, recent research showed the peak period of central nerve growth and development in animals occurred within a certain period before and after their birth, during which amplification of a large number of nerve cells of the animal's brain tissue was observed, with a large number of dendrites connecting with synapses. During this period, the central nervous system was sensitive to the peripheral microenvironment and vulnerable to stimulants affecting its normal development, which led to long-term mental and behavioral abnormalities..$^{13,14}$

Medetomidine is an agonist for central $\alpha_{2}$ adrenoceptor, which is a mixture of sinistral medetomidine and DEX, and sinistral DEX has no pharmacological effects. ${ }^{15,16}$ DEX is a dextro isomer of medetomidine. DEX is used as a sedative and analgesic in clinical setting, and its sensitivity to central $\alpha_{2}$ adrenoceptor is higher than that of medetomidine..$^{17,18}$ In order to reduce the harmful effects of general anesthesia on the nervous system, anesthetic drugs such as ketamine and propofol can be combined with DEX for producing anesthesia, and this approach has become a trend in clinical setting.
This study explored the effect of DEX on hippocampal neuronal development in neonatal rats and on the expression of molecules involved in the BDNF-TrkB signaling pathway through the separation and culturing of hippocampal neurons of the neonatal rats, in order to provide more detailed experimental evidence for the clinical application of DEX. Hippocampus is an important part of the brain tissue, and its structure and boundary are relatively clear, enabling easy dissection and separation. These advantages make it an ideal tissue for neural pharmacology experiments. In the present study, we selected neonatal rats and isolated hippocampal neuron cells for in vitro culture. Most of the neurons began to adhere to the wall within 6 hours. After culturing for 3 days, a significantly increased cell volume was observed, and the cell bodies had grown a number of protuberances. After continuous culture for 20 days, the nerve cells started to degenerate, with the appearance of granular vacuoles, degeneration of protuberances, and nuclear pyknosis. Therefore, we tried to choose early generation of cells for this experiment. MAP- 2 is a neuronal marker, and by observing the ratio of the number of MAP-2-positive labeled cells to the total number of Hoechst labeled cells, the purity of hippocampal neurons reached $90 \%$ after culturing for 7 days.

Synapse-specific protein markers such as SYN and PSD95 directly or indirectly reflected the synaptic activity, morphology changes, and synaptic biological function. Neuronal development state was indirectly shown through determining the expression of SYN and PSD95. After using different concentrations of DEX, hippocampus neuron cell viability and apoptosis were analyzed in neonatal rats. 
The differences in the expression of SYN and gap43 were detected by real-time PCR, in order to explore the developmental situation of hippocampal neurons. The results of the MTT assay showed that after treating with different doses of DEX, the neuron activity and apoptosis curve during the 2-10 days showed no statistical significance compared with the control group. There was no statistical significance in the number of positive cells between the DEX treatment groups $(1,10$, and $100 \mu \mathrm{mol} / \mathrm{L})$ and the control group. This result indicated that DEX itself had no toxicity on neural cells, which was consistent with previous studies. ${ }^{19} \mathrm{DEX}$ reduced hypoxia/reoxygenation-induced apoptosis through the inhibition of gap junctions activity by reducing Cx32 protein levels. ${ }^{20}$ Neuroprotective effect of DEX attenuates isoflurane-induced cognitive impairment through antioxidant, anti-inflammatory, and anti-apoptosis properties in aging rats. ${ }^{21}$ However, although DEX is neuroprotective at clinical doses, high cumulative doses and concentrations induce neuroapoptosis in vivo and in vitro, respectively. ${ }^{22}$

The combination of BDNF and TrkB led to a conformational change and further contributed to the phosphorylation of downstream key kinases. It has been confirmed that BDNF-TrkB signaling pathway was able to activate important pathways like MEK/ERK, PI3K/Akt, and PLC gamma 1 signaling pathways. ${ }^{23,24}$ Our experiment analyzed the effect of DEX on BDNF-TrkB signaling pathway and its downstream effector molecules associated with NMDA receptor expression and the change of phosphorylation levels. The results indicated that the BDNF expression level was positively related to DEX concentration. After treatment with $100 \mu \mathrm{mol} / \mathrm{L}$ DEX, the BDNF expression level increased significantly $(P<0.05)$. There was no correlation between the TrkB expression level and DEX treatment. There was no statistical significance among the groups with regard to the TrkB expression levels after treatment with different concentrations of DEX. The expression level of p-NMDAR showed an upward trend after DEX treatment. The variation in its background expression level exhibited no statistical significance, indicating that DEX directly or indirectly affected the activation of NMDAR, but not the expression level of NMDAR to participate in the signaling pathway.

\section{Disclosure}

The authors report no conflicts of interest in this work.

\section{References}

1. Ren Q, Ma M, Yang C, Zhang JC, Yao W, Hashimoto K. BDNF-TrkB signaling in the nucleus accumbens shell of mice has key role in methamphetamine withdrawal symptoms. Transl Psychiatry. 2015;5:e666.
2. Tian X, Hua F, Sandhu HK, et al. Effect of delta-opioid receptor activation on BDNF-TrkB vs TNF-alpha in the mouse cortex exposed to prolonged hypoxia. Int J Mol Sci. 2013;14(8):15959-15976.

3. Wei HJ, Xu JH, Li MH, et al. Hydrogen sulfide inhibits homocysteineinduced endoplasmic reticulum stress and neuronal apoptosis in rat hippocampus via upregulation of the BDNF-TrkB pathway. Acta Pharmacol Sin. 2014;35(6):707-715.

4. Mahmoud M, Mason KP. Dexmedetomidine: review, update, and future considerations of paediatric perioperative and periprocedural applications and limitations. Br J Anaesth. 2015;115(2):171-182.

5. Lee SH, Choi YS, Hong GR, Oh YJ. Echocardiographic evaluation of the effects of dexmedetomidine on cardiac function during total intravenous anaesthesia. Anaesthesia. 2015;70(9):1052-1059.

6. Lee ST, Chu K, Jung KH, et al. miR-206 regulates brain-derived neurotrophic factor in Alzheimer disease model. Ann Neurol. 2012;72(2): 269-277.

7. She YJ, Xie GT, Tan YH, et al. A prospective study comparing the onset and analgesic efficacy of different concentrations of levobupivacaine with/without dexmedetomidine in young children undergoing caudal blockade. J Clin Anesth. 2015;27(1):17-22.

8. Dolique T, Favereaux A, Roca-Lapirot O, et al. Unexpected association of the "inhibitory" neuroligin 2 with excitatory PSD95 in neuropathic pain. Pain. 2013;154(11):2529-2546.

9. Jun-HuaHu. The Protective Effect of Dexmedetomidine on Neurotoxicity of Hippocampal Neuron Cells in Rats Which Was Incubated with Propofol [dissertation]. Guangxi, People's Republic of China: Guilin Medical University; 2013.

10. Ikonomidou C, Bosch F, Miksa M, et al. Blockade of NMDA receptors and apoptotic neurodegeneration in the developing brain. Science. 1999;283(5398):70-74.

11. Jevtovic-Todorovic V, Hartman RE, Izumi Y, et al. Early exposure to common anesthetic agents causes widespread neurodegeneration in the developing rat brain and persistent learning deficits. J Neurosci. 2003; 23(3):876-882.

12. Yong-YongShi. Effect and Mechanism of Muscone on Development of Hippocampus Neurons in Neonatal Rats after Ketamine Anesthesia. Guangzhou, People's Republic of China: Southern Medical University; 2012.

13. Patterson BC, Chen Y, Sklar CA, et al. Growth hormone exposure as a risk factor for the development of subsequent neoplasms of the central nervous system: a report from the childhood cancer survivor study. J Clin Endocrinol Metab. 2014;99(6):2030-2037.

14. Hua ZL, Emiliani FE, Nathans J. Rac1 plays an essential role in axon growth and guidance and in neuronal survival in the central and peripheral nervous systems. Neural Dev. 2015;10:21.

15. Jang HS, Choi HS, Lee SH, Jang KH, Lee MG. Evaluation of the anaesthetic effects of medetomidine and ketamine in rats and their reversal with atipamezole. Vet Anaesth Analg. 2009;36(4):319-327.

16. Cruz JI, Loste JM, Burzaco OH. Observations on the use of medetomidine/ketamine and its reversal with atipamezole for chemical restraint in the mouse. Lab Anim. 1998;32(1):18-22.

17. Hui YH, Marsh KC, Menacherry S. Analytical method development for the simultaneous quantitation of dexmedetomidine and three potential metabolites in plasma. J Chromatogr A. 1997;762(1-2):281-291.

18. Ji QC, Zhou JY, Gonzales RJ, Gage EM, El-Shourbagy TA. Simultaneous quantitation of dexmedetomidine and glucuronide metabolites (G-Dex-1 and G-Dex-2) in human plasma utilizing liquid chromatography with tandem mass spectrometric detection. Rapid Commun Mass Spectrom. 2004;18(15):1753-1760.

19. Sanders RD, Sun P, Patel S, Li M, Maze M, Ma D. Dexmedetomidine provides cortical neuroprotection: impact on anaesthetic-induced neuroapoptosis in the rat developing brain. Acta Anaesthesiol Scand. 2010; 54(6):710-716.

20. Luo C, Yuan D, Yao W, et al. Dexmedetomidine protects against apoptosis induced by hypoxia/reoxygenation through the inhibition of gap junctions in NRK-52E cells. Life Sci. 2015;122:72-77. 
21. Wang X, Zhao B, Li X. Dexmedetomidine attenuates isofluraneinduced cognitive impairment through antioxidant, anti-inflammatory and anti-apoptosis in aging rat. Int J Clin Exp Med. 2015;8(10): 17281-17288.

22. Liu JR, Yuki K, Baek C, Han XH, Soriano SG. Dexmedetomidineinduced neuroapoptosis is dependent on its cumulative dose. Anesth Analg. 2016;123(4):1008-1017.
23. Luo L, Liu XL, Li J, et al. Macranthol promotes hippocampal neuronal proliferation in mice via BDNF-TrkB-PI3K/Akt signaling pathway. Eur J Pharmacol. 2015;762:357-363.

24. Semaan S, Wu J, Gan Y, et al. Hyperactivation of BDNF-TrkB signaling cascades in human hypothalamic hamartoma $(\mathrm{HH})$ : a potential mechanism contributing to epileptogenesis. CNS Neurosci Ther. 2015;21(2): $164-172$.

\section{Publish your work in this journal}

Neuropsychiatric Disease and Treatment is an international, peerreviewed journal of clinical therapeutics and pharmacology focusing on concise rapid reporting of clinical or pre-clinical studies on a range of neuropsychiatric and neurological disorders. This journal is indexed on PubMed Central, the 'PsycINFO' database and CAS, and is the official journal of The International Neuropsychiatric Association (INA). The manuscript management system is completely online and includes a very quick and fair peer-review system, which is all easy to use. Visit http://www.dovepress.com/testimonials.php to read real quotes from published authors.

Submit your manuscript here: http://www.dovepress.com/neuropsychiatric-disease-and-treatment-journal 